\title{
Three-Stage Concatenated Ultra-Wide Bandwidth Time-Hopping Spread-Spectrum Impulse Radio Using Iterative Detection
}

\author{
R. A. Riaz ${ }^{1,2}$, R. G. Maunder ${ }^{1}$, M. F. U. Butt ${ }^{1,2}$, S. X. $\mathrm{Ng}^{1}$, S. Chen ${ }^{1}$ and L. Hanzo ${ }^{1}$ \\ ${ }^{1}$ School of ECS, University of Southampton, SO17 1BJ, United Kingdom. \\ Email: \{mfub06r, rar06r, sxn, lh\}@ecs.soton.ac.uk, http://www-mobile.ecs.soton.ac.uk \\ ${ }^{2}$ Dept of EE, COMSATS Institute of Information Technology, Islamabad, 44000, Pakistan, http://ciit.edu.pk
}

\begin{abstract}
The powerful tool of EXtrinsic Information Transfer (EXIT) charts is used to design a new serially concatenated Irregular Variable Length Coded (IrVLC) and unity-rate precoded Time-Hopping (TH) Pulse Position Modulation (PPM) aided Ultra-Wide Bandwidth (UWB) Spread-Spectrum (SS) impulse radio for near-capacity operation in Nakagami-m fading channels contaminated by Partial Band Noise Jamming (PBNJ). The benefits of the 3-stage concatenation of the TH-UWB detector, the unity-rate decoder and the outer IrVLC decoder are quantified. A number of novel Variable Length Coding (VLC) codebooks having different coding rates are utilized by the IrVLC scheme for encoding specific fractions of the input source symbol stream. More explicitly, EXIT charts are employed to appropriately select these input stream fractions to shape the inverted EXIT curve of the IrVLC in order to match that of the inner decoder and hence to achieve an infinitesimally low Bit Error Ratio (BER) at near-capacity SNR values.
\end{abstract}

\section{INTRODUCTION}

UltraWideBand (UWB) communications systems are commonly defined as systems that have either more than 20 percent relative bandwidth compared to the band's center frequency or more than $500 \mathrm{MHz}$ absolute bandwidth. The large bandwidth of UWB leads to low sensitivity to fading, low interference to and from other systems along with accurate position location and ranging due to their fine time resolution [1]. Digital imaging and multimedia applications result in high data rate requirements. UWB has the capacity to fulfil the requirements of low-cost and high-speed digital indoor networks. For example, a rate of $110 \mathrm{Mbps}$ at a distance of $10 \mathrm{~m}$ and $480 \mathrm{Mbps}$ at a distance of $2 \mathrm{~m}$ can be provided with the aid of UWB links.

The pioneering work of Win and Scholtz [2] developed the concept of Time-Hopping (TH) Pulse Position Modulation (PPM) UWB impulse radio systems. In the aforementioned systems, trains of time-shifted PPM pulses are used to transmit baseband or carrierless UWB signals. One of the attractive features of TH-PPM-UWB systems is their strong multipath interference mitigating capability.

The financial support of COMSATS Institute of Information Technology, Islamabad under the auspices of Higher Education Commission, Pakistan and that of the EPSRC UK, as well as of the EU Optimix project is gratefully acknowledged.
The novel contribution of this paper is that we advance the design of TH-PPM-UWB systems with the aid of sophisticated channel coding in the interest of approaching the attainable capacity. More specifically, our near-capacity TH-PPM-UWB design exploits the fact that analogous to Irregular Convolutional Coding (ICC) [3], the family of so-called Irregular Variable Length Codes (IrVLC) [4] employs a number of component VLC codebooks having different coding rates [5] for encoding particular fractions of the input source symbol stream. The appropriate lengths of these fractions may be chosen with the aid of EXtrinsic Information Transfer (EXIT) charts [6] in order to shape the inverted EXIT curve of the IrVLC codec to ensure that it does not cross the EXIT curve of the inner channel codec. In this way, an open EXIT chart tunnel may be created even at near-capacity values of the Signal-to-Noise Ratio (SNR).

A powerful technique of enhancing the achieveable iterative detection gain is constituted by precoders, which improve the Extrinsic Information (EI) exchange between the channel decoder and the detector [7]. The precoder imposes memory upon the channel, thus resulting in an Infinite Impulse Response (IIR). Hence, Soft-Input-Soft-Output (SISO) schemes substantially benefit from the employment of low-complexity unity-rate precoders without reducing the effective throughput of the system.

As a further contribution, we extend the concept of 2stage EI exchange between the unity-rate code's decoder and an IrVLC decoder to a 3-stage scheme constituted by a unity-rate decoder, an IrVLC decoder and the TH-PPMUWB detector. The technique of EXIT charts is utilized to the best of our knowledge for the first time to design the serial concatenation of the TH-PPM-UWB detector, unity-rate decoder and an IrVLC outer decoder in order to attain a good performance even at near-capacity SNR values. We contrast the 2-stage Iterative Decoder (ID) to the 3-stage scheme and demonstrate that the 3-stage scheme outperforms the 2-stage arrangement. The practical rationale of the proposed system design procedure is that the 3-stage IrVLC-aided TH-PPMUWB scheme allows us to accurately shape the EXIT curves in order to create a marginally open EXIT tunnel, which facilitates near-capacity operation.

This paper is organised as follows. In Sec. II, our system 


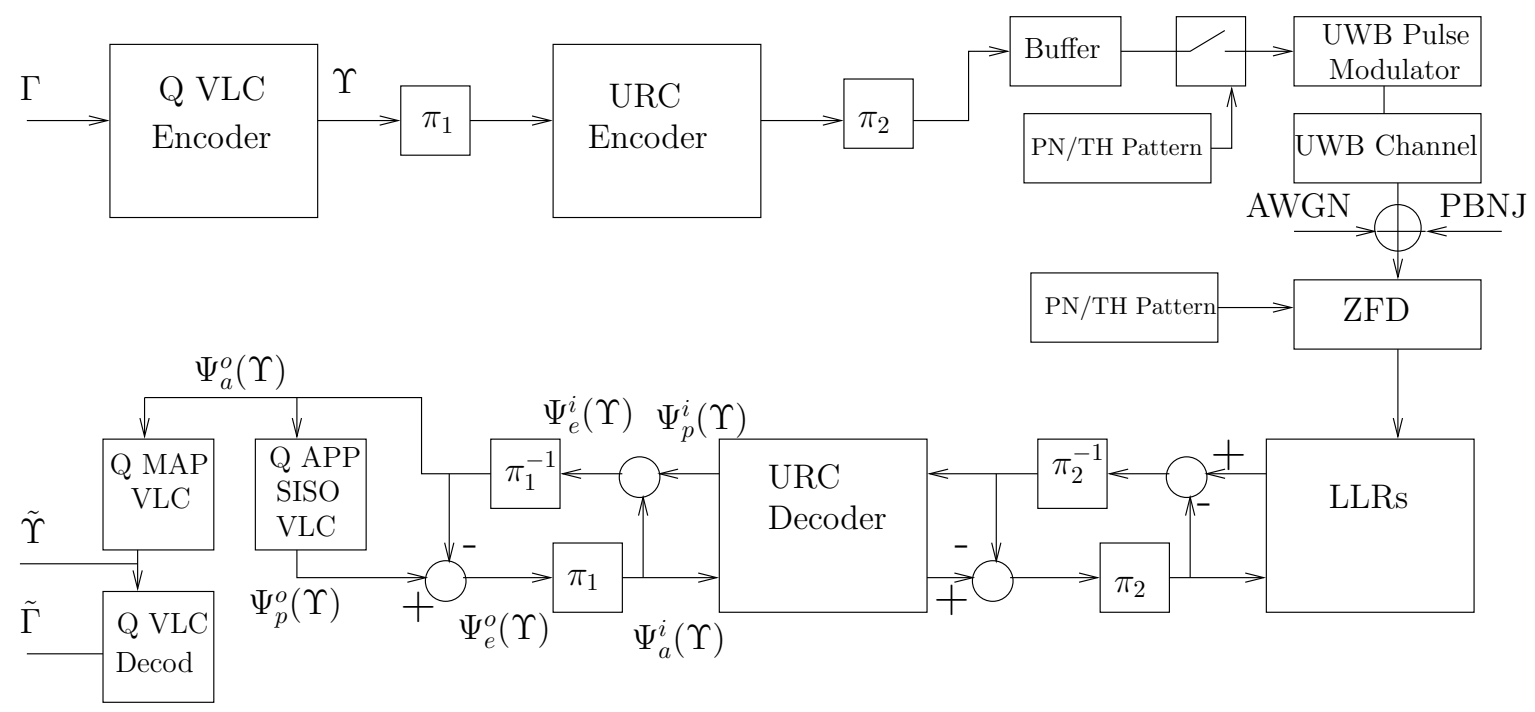

Fig. 1. Schematic of the IrVLC and VLC-based TH-PPM-UWB schemes. In the IrVLC coded scheme we have $Q=16$, whilst $Q=1$ in the VLC-coded scheme.

design philosophy is elaborated on. In Sec. III, the concept of TH-PPM-UWB transmission and detection is highlighted. In Sec. IV iterative decoding is discussed with emphasis on the associated EXIT characteristics and the soft metrics are derived. In Sec. V, both our EXIT chart and Bit Error Ratio (BER) results are discussed as a function of the IrVLC code parameters. Finally, in Sec. VI, we present our conclusions.

\section{System OVERVIEW}

Our design example considered in Fig. 1 assumes 16-ary VLC source symbol values obeying a 16-ary discrete probability density function (PDF) resulting from the Lloyd-Max (LM) quantization of independent Laplacian distributed source samples. The relative frequency range of the $16 \mathrm{VLC}$ symbols lies between 0.002 and 0.16 , when considering 4-bit LM quantization. The entropy of the 16 Laplacian distributed VLC symbols lies between 2.6 bits/symbol and 8.74 bits/symbol, with an overall source entropy of 3.5 bits per VLC symbol. The rationale of the proposed IrVLC coding scheme is that given this non-uniform probability of occurance for the 16 VLC symbols and their associated entropies, the VLC scheme is capable of data compression as well as of high-integrity detection at near-capacity SNRs.

The transmitter shown in Fig. 1 transmits the source symbol frame $\Gamma$, which comprises $J$ number of source symbols having the $K=16$-ary values $\left\{\Gamma_{j}\right\}_{j=1}^{J} \in[1 \ldots K]$. These source symbols are decomposed into $Q$ number of components $\left\{\Gamma^{n}\right\}_{n=1}^{Q}$, where we opted for $Q=16$ in the case of the IrVLC-TH-PPM-UWB scheme and $Q=1$ in the case of the regular VLC-based benchmarker scheme. The number of symbols in the source symbol frame $\Gamma$, which is decomposed into the source symbol frame component $\Gamma^{n}$ is specified as $J^{n}$, where we have $J^{1}=J$ in the case of the VLC-based scheme. By contrast, in the case of the IrVLC-based scheme, the particular values of $\left\{J^{n}\right\}_{n=1}^{Q}$ may be specifically chosen in order to shape the inverted EXIT curve of the IrVLC codec so that it does not cross the EXIT curve of the precoder.
Each of the $Q$ number of source symbol frame components $\left\{\Gamma^{n}\right\}_{n=1}^{N}$ is VLC-encoded using the corresponding codebook from the set of $Q$ number of VLC codebooks $\left\{V L C^{n}\right\}_{n=1}^{Q}$, having a range of coding rates $\left\{R^{n}\right\}_{n=1}^{Q} \in[0,1]$. The specific source symbols having the value of $k \in[1 \ldots K]$ and encoded by the specific VLC codebook $\mathbf{V L C}^{n}$ are represented by the codeword VLC ${ }^{n, k}$, which has a length of $I^{n, k}$ bits. The $J^{n}$ number of VLC codewords that represent the $J^{n}$ number of source symbols in the source symbol frame component $\Gamma^{n}$ are concatenated to provide the transmission frame component $\Upsilon^{n}=\left\{V L C^{n, \Gamma_{j^{n}}^{n}}\right\}_{j^{n}=1}^{J^{n}}$.

Depending on the specific length of the VLC codewords, the number of bits comprised by each transmission frame component $\Upsilon^{n}$ will typically vary slightly from frame to frame. In order to facilitate the VLC decoding of each transmission frame component $\Upsilon^{n}$, it is necessary to explicitly convey its length $I^{n}=\sum_{j^{n}=1}^{J^{n}} I^{n, s_{j^{n}}^{n}}$ to the receiver with the aid of side information. Furthermore, this highly error-sensitive side information must be reliably protected against transmission errors. This may be achieved using a low rate block code or repetition code, for example. For the sake of avoiding obfuscating details, this is not shown in Fig. 1.

The $Q$ transmission frame components $\left\{\Upsilon^{n}\right\}_{n=1}^{Q}$ encoded by the different IrVLC component codes are concatenated at the transmitter, as shown in Fig. 1. The resultant transmission frame $\Upsilon$ has a length of $\sum_{n=1}^{Q} I^{n}$ bits. Following the bit interleaver $\pi_{1}$, the transmission frame $\Upsilon$ is precoded and then interleaved again by the bit interleaver $\pi_{2}$. The interleaved bits are sent to the buffer depicted in Fig. 1. These bits are transmitted by the TH-PPM modulator. In TH-PPMUWB systems the transmitted TH-UWB signal conveying a specific M-ary symbol is constituted by several frames. Let one symbol invokes $L$ number of frames. The frame duration $T_{F}$ is related to the symbol interval $T_{S}$ by $T_{S}=L T_{F}$. Furthermore, the modulating symbol-dependent desired time shift $T_{C H}$ is generated by a unique, user-specific $\mathrm{TH}$ code. As depicted in Fig. 1, we have used a pseudo-noise (PN) 
generator for generating these $\mathrm{TH}$ sequences. The details of UWB transmission will be discussed in the next section.

The achieveable performance of the proposed TH-PPMUWB system will be evaluated using an UWB multipath channel model based on indoor channel measurements between 3.1 GHZ to $10.6 \mathrm{GHz}$ over a range of less than 10 meters. The UWB channel Impulse Response (CIR) taps typically obey a Nakagami distribution. The transmitted signal is also corrupted both by Additive White Gaussian Noise (AWGN) and Partial Band Noise Jamming (PBNJ) having single-sided power spectral densities of $N_{0}$ and $N_{J}$, respectively.

The receiver schematic is also shown in Fig. 1, where the Zero Forcing Detector (ZFD) [8] is used to estimate the data $\hat{\mathrm{d}}$. The ZFD has the capability of combating the effects of both Multiple Access Interference (MAI) and Inter-Symbol Interference (ISI) at the expense of enhancing the noise at low SNRs. After the ZFD, the corresponding symbol probabilities and log-likelihood ratios (LLRs) are computed, as detailed in Sec. IV, which are then fed to the unity-rate decoder of Fig. 1.

\section{UWB TRANSMISSION AND DETECTION}

\section{A. TH PPM UWB}

The TH-PPM-UWB signal transmitted by the schematic of Fig. 2 is given by [9]

$$
g(t)=\sum_{n=-\infty}^{\infty} \phi\left(t-n T_{F}-T_{P P_{n}}-T_{C H_{n}}\right),
$$

where $\phi(t)$ is the signalling pulse shape, $T_{F}$ is the frame duration, $T_{P P_{n}}$ is the PPM-related shift in the pulse position, either forward or backward with respect to the nominal signalling instant to represent the binary stream, $T_{C H_{n}}$ is the time shift based on the unique time hopping code of a specific user, where the code repeats after a certain interval. The pulse repetition frequency (PRF) is the reciprocal of $T_{F}$. The frame duration $T_{F}$ is typically on the order of 1000 times higher than the actual pulse width in order to avoid any inter-symbol interference imposed by multipath propagation.

In Fig. 2 we show the output of a simple TH aided binary PPM UWB transmitter. The schematic characterises a singleuser scenario, which can be readily extended to the multi-user scenario by using different time-hopping PN codes for the different users, as seen in Fig. 1.

\section{B. Channel Model}

The CIR model ratified by the IEEE 802.15.3 standard and considered here can be expressed as [10]

$$
h(t)=\sum_{r=1}^{R} h_{r} e^{j \varphi_{r}} \delta\left(t-r T_{\varphi}\right),
$$

where $R$ represents the number of resolvable paths, while $h_{r}$ and $\varphi_{r}$ are the gain and phase of the $r$ th resolvable multipath component. Furthermore, $r T_{\varphi}$ represents the corresponding delay of the $r$ th multipath component.

As shown in [11], [12] the CIR taps of the UWB channel follow the Nakagami-m distribution, which has been validated by using the Kolmogorov-Smirnov testing at a significance

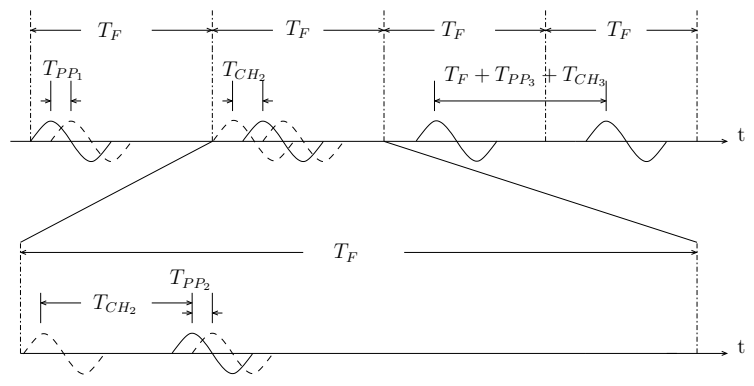

Fig. 2. A binary TH-PPM system output.

level of $1 \%$. More explicitly, the fading gains $h_{r}$ obey an independent Nakagami-m distribution with a probability density function (PDF) of the envelop $c$ given by [13]

$$
P_{h_{r}}(c)=\frac{2 m_{r}^{m_{r}} r^{2 m_{r}-1}}{\Gamma\left(m_{r}\right) \Omega_{r}^{m_{r}}} e^{\frac{-m_{r} c^{2}}{\Omega_{r}}}, \quad c>0
$$

where $m_{r}$ is the Nakagami fading parameter defined as:

$$
m_{r}=\frac{E^{2}\left[c^{2}\right]}{\operatorname{var}\left(c^{2}\right)},
$$

while the parameter $\Omega_{r}$ is $E\left[c^{2}\right]$. Furthermore, the moments of the random variable $c$ are given by:

$$
E\left[c^{v}\right]=\frac{\Gamma\left(m_{r}+\frac{v}{2}\right)}{\Gamma\left(m_{r}\right)}\left(\frac{\Omega}{m_{r}}\right)^{\frac{v}{2}} .
$$

Finally, $\Gamma(\cdot)$ is the gamma function defined as

$$
\Gamma\left(m_{r}\right)=\int_{0}^{\infty} x^{m_{r}-1} e^{(-x)} d x .
$$

We assume in our analysis that the phase rotation of the fading channel is uniformly distributed in $[0,2 \pi]$. Let us now consider the receiver structure.

\section{Receiver Model}

Zero Forcing Detector The discretized received composite signal can be represented in matrix form as [8]:

$$
\mathbf{y}=\mathbf{A d}+\mathbf{n},
$$

where $\mathbf{n}$ is the noise sequence, which has a covariance matrix of $\mathbf{R}_{n}=E\left[n n^{H}\right], \mathbf{A}$ is the overall system matrix and $\mathbf{d}$ is the user data vector. The data estimates at the output of the ZFD are:

$$
\hat{\mathbf{d}}_{\mathbf{Z F D}}=\underbrace{\mathbf{d}}_{\text {Data }}+\underbrace{\left(\mathbf{A}^{\mathbf{H}} \mathbf{R}_{\mathbf{n}}^{-\mathbf{1}} \mathbf{A}\right)^{-1} \mathbf{A}^{\mathbf{H}} \mathbf{R}_{\mathbf{n}}^{-\mathbf{1}} \mathbf{n}}_{\text {Noise }} .
$$

If it is assumed in $\mathrm{Eq} \mathrm{(8)} \mathrm{that} \mathbf{n}$ consists of noise samples that are zero-mean Gaussian variables having a variance of $\sigma^{2}$, then the corresponding covariance matrix is $\mathbf{R}_{n}=\sigma^{2} I$, where $\mathrm{I}$ is the identity matrix. Hence Eq (8) can be reduced to

$$
\left.\hat{\mathbf{d}}_{\mathbf{Z F D}}\right|_{\mathbf{R}_{n}=\sigma^{2} I}=\left(\mathbf{A}^{\mathbf{H}} \mathbf{A}\right)^{-\mathbf{1}} \mathbf{A}^{\mathbf{H}} \mathbf{y} .
$$

It is evident from Eqs (8) and (9) that the ZFD decorrelates the MAI and ISI and forces them to zero. However, the removal of the ISI and MAI is performed at the expense of potential noise enhancement. 


\section{ITERATIVE DECODING}

The conditional probability of the $j$ th transmitted symbol $\Theta_{j}$, where $j=0, \ldots, J-1$, given the signal $\hat{\mathbf{d}}=$ $\left[d_{0}, d_{1}, \ldots, d_{J-1}\right]$, which represents the set of $J$ outputs of the ZFD seen Fig. 1 is given by

$$
P\left(\Gamma_{j} \mid \hat{\mathbf{d}}\right)=\frac{p\left(\hat{\mathbf{d}} \mid \Gamma_{j}\right) P\left(\Gamma_{j}\right)}{p(\hat{\mathbf{d}})},
$$

where $p\left(\hat{\mathbf{d}} \mid \Gamma_{j}\right)$ is the PDF of the received signal $\hat{\mathbf{d}}$, given that $\Gamma_{j}$ is transmitted. Furthermore, $P\left(\Gamma_{j}\right)$ is the a priori probability of the symbol $\Gamma_{j}$, while $p(\hat{\mathbf{d}})=\sum_{j=0}^{J-1} p\left(\hat{\mathbf{d}} \mid \Gamma_{j}\right) P\left(\Gamma_{j}\right)$ is the probability of receiving the signal set $\hat{\mathbf{d}}$. At the first iteration we have $P\left(\Gamma_{j}\right)=1 / J$ for all the transmitted symbols, since no a priori information is available and $\operatorname{PDF} p\left(\hat{\mathbf{d}} \mid \Gamma_{j}\right)$ uniquely determines the statistics required for estimating the probability $P\left(\Gamma_{j} \mid \hat{\mathbf{d}}\right)$. The expression of $p\left(\hat{\mathbf{d}} \mid \Gamma_{j}\right)$ is given by

$$
p\left(\hat{\mathbf{d}} \mid \Gamma_{j}\right)=f_{d_{j}}\left(v_{j} \mid \Gamma_{j}\right) \prod_{x=0, x \neq j}^{J-1} f_{d_{n}}\left(v_{x} \mid \Gamma_{j}\right),
$$

where $f_{d_{n}}\left(v_{x} \mid \Gamma_{j}\right)$ represents the PDF of the $x$ th detector value, $x=0,1, \ldots, J-1$, given that $\Gamma_{j}$ is transmitted. Then the simplified expression of $p\left(\hat{\mathbf{d}} \mid \Gamma_{j}\right)$ becomes

$$
p\left(\hat{\mathbf{d}} \mid \Gamma_{j}\right)=\exp \left(\frac{v_{j} \gamma_{h}}{1+\gamma_{h}}\right),
$$

where $\gamma_{h}=b R E_{b} /\left(N_{0} L\right)$ is the SNR per hop, $R$ is the code rate, $E_{b}$ is the transmitted energy per bit and $b=\log _{2} M$ is the number of bits per symbol. The corresponding Log-Likelihood Ratios (LLRs) can be computed [6] from Eqs. (11) and (12).

The derivation of the soft information from the received signal is different for the 2 and 3 -stage serial concatenated schemes. In the first case, the a posteriori probability (APP) Soft-Input-Soft-Output (SISO) unity-rate decoder and the outer decoder exchange EI in order to perform Iterative Detection (ID), both invoking the Bahl-Cocke-Jelinek-Raviv (BCJR) algorithm using bit-based trellises. Again, we refer to this ID-aided configuration as the 2-stage scheme. Alternatively, the system may be modified so that the TH-PPMUWB detector, the unity-rate inner decoder and the IrVLC outer decoder exchange their EI, as shown in Fig. 1. We refer to this arrangement as the 3-stage scheme. The 3-stage scheme requires the additional interleaver $\pi_{2}$ between the precoder and the TH-PPM-UWB detector of Fig. 1.

Let us now highlight the EI characteristics of the ID process using EXIT curves [6]. All EI evaluations involved the histogram based approximation of the true EI distribution [6]. Note that in Fig. $1 \Psi(\cdot)$ denotes the LLRs of the bits concerned, where the superscript $i$ indicates the inner decoder (or detector), while $o$ corresponds to the outer decoder. Additionally, the specific subscripts denote the dedicated role of the LLRs, with $a, p$ and $e$ indicating a priori, a posteriori and extrinsic information, respectively. Moreover, unless otherwise stated, we employ the following parameter values: source symbol frame length of $J=80,000$, outer code rate of $R=0.5$, TH-PPM-UWB spreading factor of $M=16$ and diversity order of $L=3$.
The TH-PPM-UWB detector yields low-gradient EXIT curves for all values of the spreading factor $M$, which do not reach the $(1,1)$ point of the EXIT chart. When the SNR is increased, the EXIT curves are shifted upwards in the EXIT plane and hence an open EXIT tunnel and an arbitrarily low BER may be achieved. However, this would be attained at the cost of having a relatively large area between the detector's and decoder's EXIT curves, implying that the scheme operates far from capacity, as detailed and justified in [4]. However, the precoder renders the channel to appear recursive [7], resulting in an IIR and hence in steeper rising EXIT curves than the stand-alone detector. Moreover, in contrast to the demodulator, the unity-rate decoder's EXIT curves do indeed reach the $\left(I_{a}, I_{e}\right)=(1,1)$ point, implying that the precoder allows the ID-aided scheme to converge to an arbitrarily low BER.

Let us now employ EXIT charts in order to design the 3stage scheme of Fig. 1. Ideally, this would require 3-D EXIT charts for depicting the evolution of the EI at the output of all the three serially concatenated components. However, a simpler and almost equally effective method of investigating this 3-stage EI exchange was proposed in [14], which implies treating the detector and the unity-rate inner decoder as a single composite-module, which may employ a number of inner iterations between the two components. This effectively allows us to analyze the 3-stage concatenation as a 2-stage one using 2-D EXIT charts.

In these investigations the composite-module invokes a single iteration of EI exchange between the detector and the unity-rate decoder, because using more than one iterations between these two blocks provides no substantial benefit. Hence, when the 3 -stage scheme of Fig. 1 is employed, each ID iteration involves a single iteration between the detector and the unity-rate decoder, followed by a single iteration between the unity-rate inner decoder and the outer IrVLC decoder. Moreover, the 3-stage scheme employed a precoder of memory 1 , while the 2-stage scheme involved a precoder of memory 3 , since it was found that using these precoder memories attractive EXIT characteristics were achieved at the cost of a low complexity.

Since $Q$ number of separate VLC encoders are employed in the TH-PPM-UWB transmitter, $Q$ number of separate VLC decoders have to be used in the corresponding receiver seen in Fig. 1. In parallel to the composition of the bit-based transmission frame $\Upsilon$ from $Q$ number of VLC source symbols, the a priori LLRs $\Psi_{a}^{o}(\Upsilon)$ are decomposed into $Q$ number of components, as shown in Fig. 1. This is achieved with the aid of the explicit side information that we assume for conveying the slightly varying number of bits $I^{n}$ of each transmission frame component $\Upsilon^{n}$. Each of the $Q$ number of VLC decoders is provided with the a priori LLR sub-frame $\Psi_{a}^{o}\left(\Upsilon^{n}\right)$ and in response it generates the a posteriori LLR sub-frame $\Psi_{p}^{o}\left(\Upsilon^{n}\right), n \in[1 \ldots Q]$. These a posteriori LLR subframes are concatenated in order to provide the a posteriori LLR frame $\Psi_{p}^{o}(\Upsilon)$, as shown in Fig. 1 .

During the final decoding iteration, $Q$ number of bit-based Maximum Aposteriori Probability (MAP) VLC sequence estimation processes are invoked instead of single-class APP SISO VLC decoding, as shown in Fig. 1. In this case, each 
transmission frame component $\Upsilon^{n}$ is estimated from the corresponding a priori LLR frame component $\Psi_{a}^{o}\left(\Upsilon^{n}\right)$. The resultant transmission frame component estimates $\tilde{\Upsilon}^{n}$ may be concatenated to provide the transmission frame estimate $\tilde{\Upsilon}$. Additionally, the transmission frame component estimates $\tilde{\Upsilon}^{n}$ may be VLC decoded to provide the source symbol frame component estimates $\tilde{\Gamma}^{n}$.

In the next section we detail the design of our three-stage concatenated TH-PPM-UWB scheme with the aid of EXIT chart analysis.

\section{System PARAmEters AND Simulation Results}

We have used $Q=16$-component VLC codebooks $\left\{V L C^{n}\right\}_{n=1}^{Q}$ having approximately equally spaced coding rates in the range of $[0.2,0.95]$ in the TH-PPM-UWB scheme. In each case, we employ a Variable Length Error Correcting (VLEC) codebook [5] that is tailored to the source symbol values' probabilities of occurrence. Again, these codes compress the unequal probability input data symbols for the sake of achieving a near-entropy source-rate and a low error probability at near-capacity SNRs. By contrast, in the VLC benchmarker scheme, we employ just $Q=1$ VLC codebook, which is identical to the VLC codebook $V L C^{10}$ of the IrVLC scheme, having a coding rate of $R=0.5$. Note that this coding rate results in an average interleaver length of $J \cdot \frac{E}{R}$ bits.

Fig. 3 and Table I depicts the inverted EXIT curves that characterise the bit-based APP SISO VLC decoding of the above-mentioned VLC codebooks, together with the rate-1 decoder's EXIT curves at $\frac{E_{b}}{N_{0}}$ values of 5.9 and 5.8 [dB], assuming an uncorrelated Nakagami-m fading channel contaminated by PBNJ characterised by $\frac{E_{b}}{N_{J}}=10 \mathrm{~dB}$ and a jamming factor of $\rho=0.1$.

TABLE I

VLC PARAMETERS AND CODE RATES

\begin{tabular}{|l|l|}
\hline 1. $V L C^{1}, R^{1}=0.95, J^{1}=0$ & $11 . V L C^{11}, R^{11}=0.45, J^{11}=0$ \\
\hline 2. $V L C^{2}, R^{2}=0.89, J^{2}=0$ & $12 . V L C^{12}, R^{12}=0.4, J^{12}=0.5 J$ \\
\hline 3. $V L C^{3}, R^{3}=0.85, J^{3}=0$ & $13 . V L C^{13}, R^{13}=0.35, J^{13}=0$ \\
\hline 4. $V L C^{4}, R^{4}=0.8, J^{4}=0.2 J$ & $14 . V L C^{14}, R^{14}=0.30, J^{14}=0$ \\
\hline 5. $V L C^{5}, R^{5}=0.75, J^{5}=0$ & 15. $V L C^{15}, R^{15}=0.25, J^{15}=0$ \\
\hline 6. $V L C^{6}, R^{6}=0.70, J^{6}=0.12 J$ & 16. $V L C^{16}, R^{16}=0.20, J^{16}=0$ \\
\hline 7. $V L C^{7}, R^{7}=0.65, J^{7}=0$ & 17. IrVLC $=0.5$ \\
\hline 8. $V L C^{8}, R^{8}=0.6, J^{8}=0.2 J$ & 18. Precoder $S N R=5.9 d B$ \\
\hline 9. $V L C^{9}, R^{9}=0.55, J^{9}=0$ & 19. Precoder $S N R=5.8 d B$ \\
\hline 10. $V L C^{10}, R^{10}=0.5, J^{10}=0.2 J$ & 20. EXIT Open Tunnel $5.9 d B$ \\
\hline
\end{tabular}

The inverted EXIT curve of the IrVLC scheme is also shown in Fig. 3. This was obtained as the appropriately weighted superposition of the $Q=16$ component VLC codebooks' inverted EXIT curves, where the weight applied to the inverted EXIT curve of the component VLC codebook $\mathbf{V L C}^{\mathbf{n}}$ is proportional to the specific number of source symbols employed for encoding $J^{n}$ [3]. Using the approach of [3], the values of $\left\{J^{n}\right\}_{n=1}^{Q}$ given in Fig. 3 were designed so that the IrVLC coding rate matches that of our regular VLC benchmarker scheme, namely 0.5. Furthermore, we ensured that the inverted IrVLC EXIT curve did not cross the unityrate decoder's EXIT curve at $\frac{E_{b}}{N_{0}}$ of $5.9 \mathrm{~dB}$. We note that only 4 of the 16 VLC components were indeed activated by

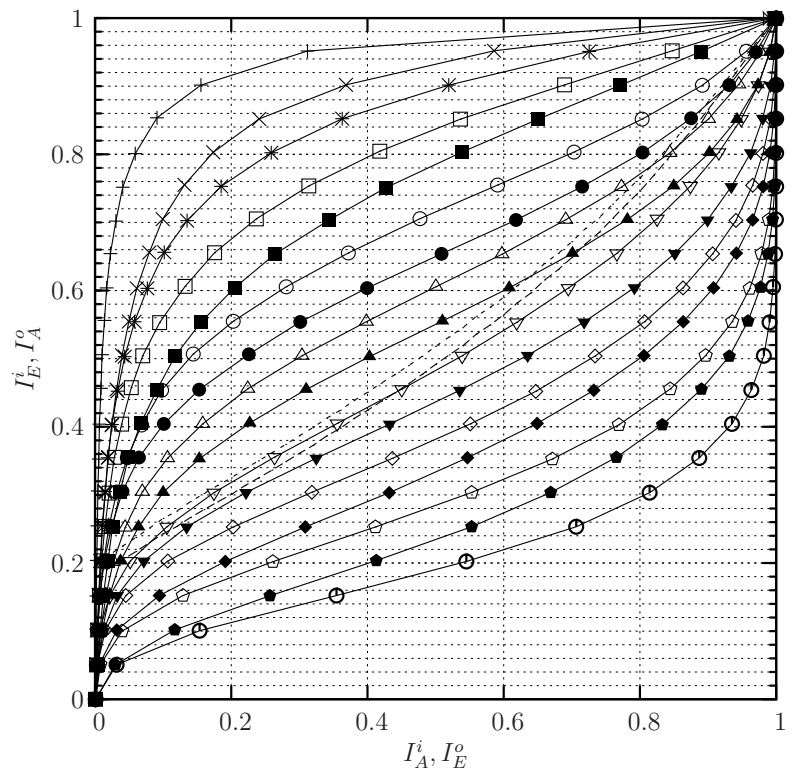

$$
\begin{aligned}
& \text { Table-I:1 — Table-I:6 ○- Table-I:11 \-Table-I:16 - } \\
& \text { Table-I: } 2 \longrightarrow \text { Table-I: } 7 \multimap \text { Table-I:12 } \prec \text { Table-I:18 } \\
& \text { Table-I: } 3 \longrightarrow \text { Table-I:8 } \triangle \text { Table-I:13 } \longrightarrow \text { Table-I:19 ----- } \\
& \text { Table-I:4 } \square \text { Table-I:9 } \leadsto \text { Table-I:14 ๑ } \\
& \text { Table-I:5 - Table-I:10 } \nabla \text { Table-I:15 • }
\end{aligned}
$$

Fig. 3. Inverted VLC EXIT curves and unity rate decoder EXIT curves, assuming an interference-free, uncorrelated Nakagami-m fading channel.

the algorithm of [3] in order to encode a non-zero number of source symbols. As shown in Fig. 3, the presence of an open EXIT chart tunnel implies that an infinitesimally low BER may be achieved by the TH-PPM-UWB scheme for $\frac{E_{b}}{N_{0}}$ values above $5.9 \mathrm{~dB}$. By contrast, an open EXIT chart tunnel is not afforded for $\frac{E_{b}}{N_{0}}$ values below $5.8 \mathrm{~dB}$ in the case of the benchmarker VLC-based scheme. Analogous to the IrVLC design of Fig. 3, we have also designed IrVLC codes for both the 2-stage and 3-stage ID scheme, assuming various jamming scenarios in Nakagami-m fading channels. The code rates of the IrVLC's component codes used in our simulations are $[0.95,0.89,0.85,0.8,0.75,0.7,0.65,0.6,0.55,0.5,0.45,0.4$, $0.35,0.3,0.25,0.2]$.

Fig. 4 provides the BER performance of both the 2-stage and the 3 -stage schemes versus $\frac{E_{b}}{N_{J}}$, assuming $\frac{E_{b}}{N_{0}}=10 \mathrm{~dB}$ and $\rho=0.5$. It becomes explicit from Fig. 4 that the 3 -stage scheme yields an improvement of nearly $3 \mathrm{~dB}$ over the 2-stage IrVLC. Both schemes result in a superior performance compared to the system operating without the precoder, since the latter encounters an error floor. The performance gain achieved by the 3-stage scheme is at the expense of a slightly higher complexity imposed by the extra interleaver and decoder, as shown in Fig. 1. Finally, Fig. 5 portrays the EXIT curves of the IrVLC and unity-rate decoders as well as the corresponding decoding trajectory, assuming an interference free, uncorrelated Nakagami-m fading channel for an SNR of 5.9dB.

\section{CONCLUSION}

In this treatise we have investigated the serial concatenation of IrVLC coding with a TH-PPM-UWB scheme operating in 


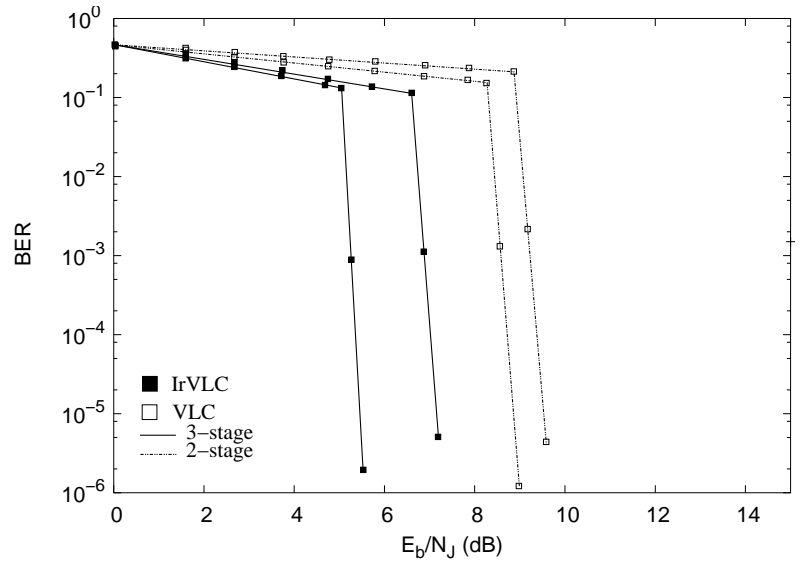

Fig. 4. BER versus $\frac{E_{b}}{N_{J}}$ performance of the 2-stage and 3-stage VLC and IrVLC based schemes in jammed, uncorrelated Nakagami-m fading channels assuming $\frac{E_{b}}{N_{o}}=10 \mathrm{~dB}$ and $\rho=0.5$ with 40 iterations.

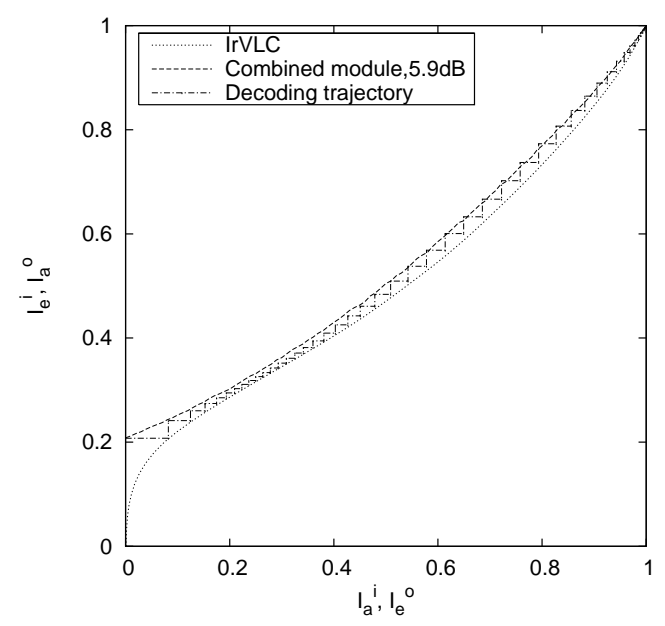

Fig. 5. IrVLC and Unity-rate decoders EXIT curves as well as decoding trajectory, assuming jammed, uncorrelated Nakagami-m fading channels with $\rho=0.1$.

a Nakagami-m fading channel in conjunction with PBNJ. In addition to this 2-stage scheme we have also investigated the 3stage concatenation of the TH-PPM-UWB arrangement with a unity-rate decoder and the outer IrVLC decoder using 2D EXIT charts. Consequently, we noted that the precoderaided scheme yields a gain of about $6.9 \mathrm{~dB}$ over the system operating without a precoder, since an open EXIT chart tunnel may be created at low SNR values. Moreover, the 3-stage scheme performs $2.9 \mathrm{~dB}$ better than the corresponding 2-stage scheme.

\section{REFERENCES}

[1] M. Z. Win and R. A. Scholtz, "On the energy capture of ultrawide bandwidth signals in dense multipath environments," IEEE Communications Letters, vol. 2, pp. 245-247, Sept. 1998.

[2] M. Z. Win and R. A. Scholtz, "Impulse radio: how it works," IEEE Communications Letters, vol. 2, pp. 36-38, Feb. 1998.

[3] M. Tüchler and J. Hagenauer, "EXIT charts of irregular codes," in Conference on Information Sciences and Systems, (Princeton, NJ), pp. 748-753, March 2002.
[4] R. G. Maunder, J. Wang, S. X. Ng, L. L. Yang, and L. Hanzo, "On the performance and complexity of irregular variable length codes for near-capacity joint source and channel coding," IEEE Transactions on Wireless Communications, vol. 7, pp. 1338-1347, Apr. 2008.

[5] V. Buttigieg and P. G. Farrell, "Variable-length error-correcting codes," IEE Proceedings on Communications, vol. 147, pp. 211-215, August 2000.

[6] S. ten Brink, "Convergence of iterative decoding," IEEE Transactions on Communications, vol. 49, pp. 1727 - 1737, October 2001.

[7] R. Y. S. Tee, S. X. Ng, and L. Hanzo, "Precoder-aided iterative detection assisted multilevel coding and three-dimensional EXIT-chart analysis," in IEEE Wireless Communications and Networking Conference, 2006. WCNC 2006, vol. 3, pp. 1322-1326, April 2006.

[8] L. Hanzo, L.-L. Yang, E.-L. Kuan, and K. Yen, Single- and Multi-Carrier DS-CDMA: Multi-User Detection, Space-Time Spreading, Synchronisation, Networking and Standards. New York: John Wiley and Sons, England, 2003.

[9] R. A. Riaz, M. El-Hajjar, Q. Z. Ahmed, S. X. Ng, S. Chen, and L. Hanzo, "Convergence Analysis of Iteratively Detected Time Hopping and DSCDMA Ultrawide Bandwidth Systems by EXIT Charts," in Proceedings of the IEEE Vehicular Technology Conference, 2008., pp. 1127 - 1131, May 2008.

[10] H. Sato and T. Ohtsuki, "Frequency domain channel estimation and equalisation for direct sequence ultra wideband (DS-UWB) system," IEE Proceedings-Communications, vol. 153, pp. 93-98, Feb. 2006.

[11] A. F. Molisch, "Ultrawideband propagation channels-theory, measurement, and modeling," IEEE Transactions on Vehicular Technology, vol. 54, pp. 1528-1545, Sept. 2005.

[12] R. A. Riaz, M. F. U. Butt, S. Chen, and L. Hanzo, "Generic z-domain discrete-time transfer function estimation for ultra-wideband systems," Electronics Letters, vol. 44, pp. 1491-1492, Dec. 2008.

[13] M. D. Yacoub, J. E. V. Bautistu, and L. Guerra de Rezende Guedes, "On higher order statistics of the nakagami-m distribution," IEEE Transactions on Vehicular Technology, vol. 48, pp. 790-794, May 1999.

[14] J. Wang, S. X. Ng, A. Wolfgang, L.-L. Yang, S. Chen, and L. Hanzo, "Near-capacity three-stage MMSE turbo equalization using irregular convolutional codes," in International Symposium on Turbo Codes, (Munich, Germany), April 2006. 